\title{
Gallipoli nel paesaggio fortificato del Mediterraneo
}

\author{
Gallipoli in the Mediterranean fortified landscape
}

\section{Giovanna Cacudi}

Soprintendenza Archeologia Belle Arti e Paesaggio per le province di Brindisi Lecce e Taranto, Lecce, Italy, giovanna.cacudi@beniculturali.it

\begin{abstract}
The paper, through the study of the main maps and historical imagery, intends to describe through pictures the fortified city of Gallipoli alongside the evolution of the Mediterranean coastal landscape and, specifically of the Salento peninsula. Starting from the analysis of the Piri Reis map, a specific selection of depictions shows how the city with its castle, its fortifications and its ancient port overlooking the Ionic coast, was always identified as territorial landmark; it has been geographically recognizable since the first maps and views, both for the structure intended to protect from incursions from the sea and for the commercial exchanges with other mediterranean countries. From the analysis of images made at different times and for different purposes, it appears that the Gallipoli has always been seen as a center of primary importance in the fortified coastal landscape, like it still are today. In spite of the progressive urban growth, the phenomena of conurbation and the transformations of the coasts caused by coastal anthropization remain fully recognizable in their original structure and in their inseparable relationship with the marine and terrestrial landscape.
\end{abstract}

Keywords: Gallipoli, fortified town, landscape.

\section{Scoprire Gallipoli}

Possiamo solo immaginare la curiosità con cui il giovane Piri Re'is ${ }^{1}$ abbia un giorno scrutato l'orizzonte nel tentativo di scorgere, per la prima volta, il profilo della città di Gallipoli, uguale nel nome alla sua città natale sullo stretto dei Dardanelli, e supporre che nel suo percorrere in lungo e in largo il Mediterraneo ci sia, forse più volte, ritornato: ne sono indizio la cura con cui, anni dopo e in età matura, nel realizzare il suo Kitab i bahriye, il Libro del mare, ha voluto rappresentare la città-isola con un rilievo e una cura dei particolari che supera di gran lunga altre città di mare della costa dell'Italia meridionale, compresa la stessa Taranto. Datato al 1520-1525, il Libro del mare è un portolano ${ }^{2}$ destinato ai capitani delle flotte barbaresche operanti nel Medi- terraneo. Nella sua rappresentazione -la prima di cui abbiamo notizia-, Gallipoli è già perfettamente riconoscibile negli elementi che costituiscono la sua struttura urbana attuale, con la cinta muraria punteggiata di torri e bastioni, il Castello e il ponte di collegamento alla terraferma.

All'interno del perimetro difensivo spiccano le emergenze architettoniche del castello, della cattedrale, della chiesa e convento di San Francesco d'Assisi all'epoca già sicuramente esistente ${ }^{3}$, allineati lungo un asse che attraversa la città. Il complesso conventuale dei Domenicani e la chiesa confraternale del Carmine, con cui possono identificarsi gli altri due edifici religiosi che si vedono nella mappa, sono entrambi documentati al XVI secolo ${ }^{4}$ (Fig. 1, tratta alla tavola pub- 
blicata da Capone Editore in Imago Apuliae [Cartella di 16 stampe a colori]). In una edizione differente del Kitab $i$ bahriye conservata al The Walters Art Museum (Piri Re'is, 1525), oltre al Castello ed alla cattedrale compaiono solo due edifici religiosi: il complesso conventuale di S. Francesco d'Assisi e quello dei Domenicani (Fig. 2).

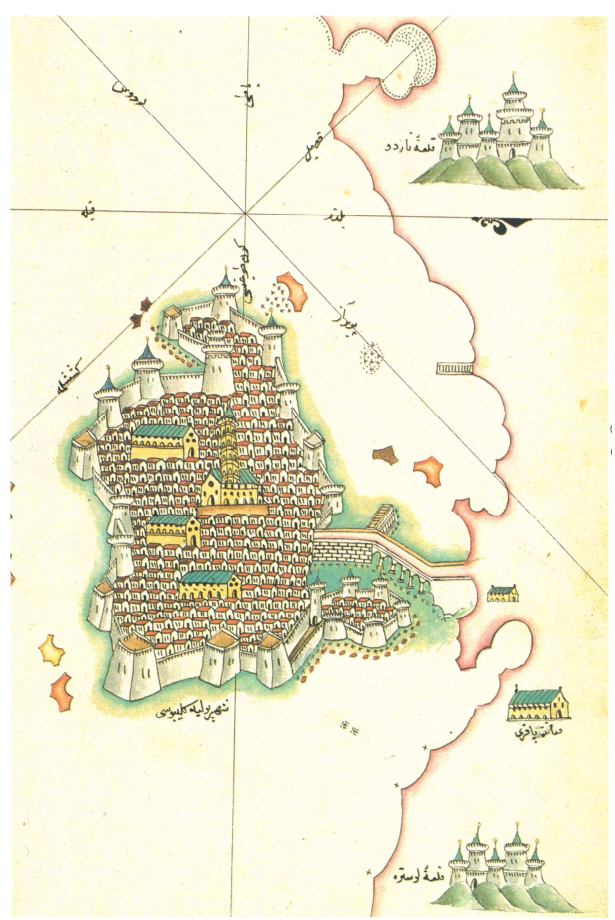

Fig. 1. Gallipoli nella tavola del Libro del mare di Piri Re'is pubblicata da Capone Editore.

Sulla costa, all'altro estremo del ponte, si riconosce la chiesa della Madonna del Canneto, ricostruita nel 1696 sulle vestigia di un preesistente edificio del XIII-XIV secolo (Mario, 2002); nella Mappa pubblicata da Capone, si identificano le città di Ugento posta a sud di Gallipoli e Nardò posta a nord (la rappresentazione ha infatti il sud orientato verso l'alto). Nella tavola conservata presso il "The Walters Art Museum" compare tra i due un terzo abitato.

Più realisticamente, il rilievo dato dal Kitab $i$ bahriye alla città è dovuta all'importanza militare e commerciale rivestita da Gallipoli a partire dal XVI secolo.

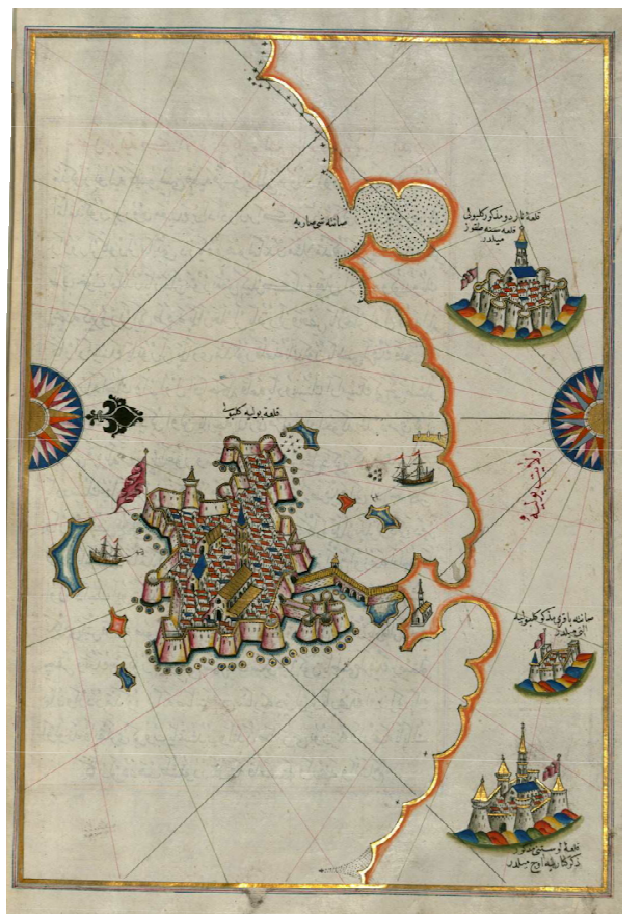

Fig. 2. La stessa rappresentazione nell'edizione conservata presso il "The Walters Art Museum".

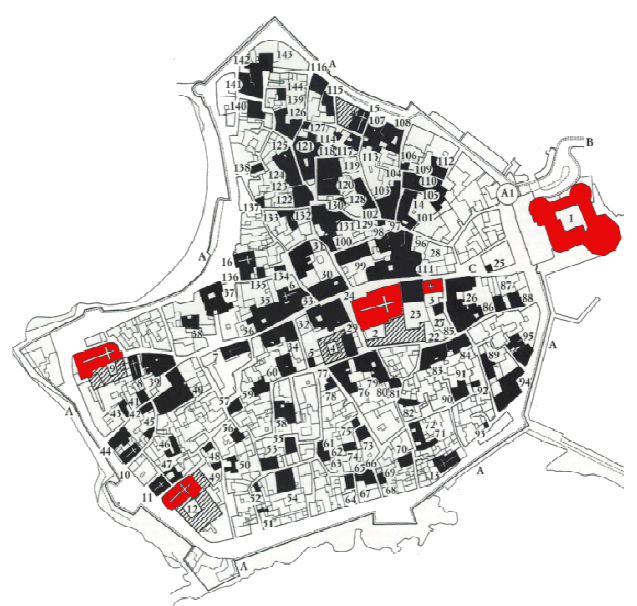

Fig. 3. Pianta attuale di Gallipoli. In evidenza il Castello, la Cattedrale e il Convento di S. Francesco d'Assisi, in allineamento tra loro. In basso, il complesso dei Domenicani; dietro la Cattedrale, la Chiesa confraternale del Carmine (da Cazzato, et al., 2015, con modifiche). 


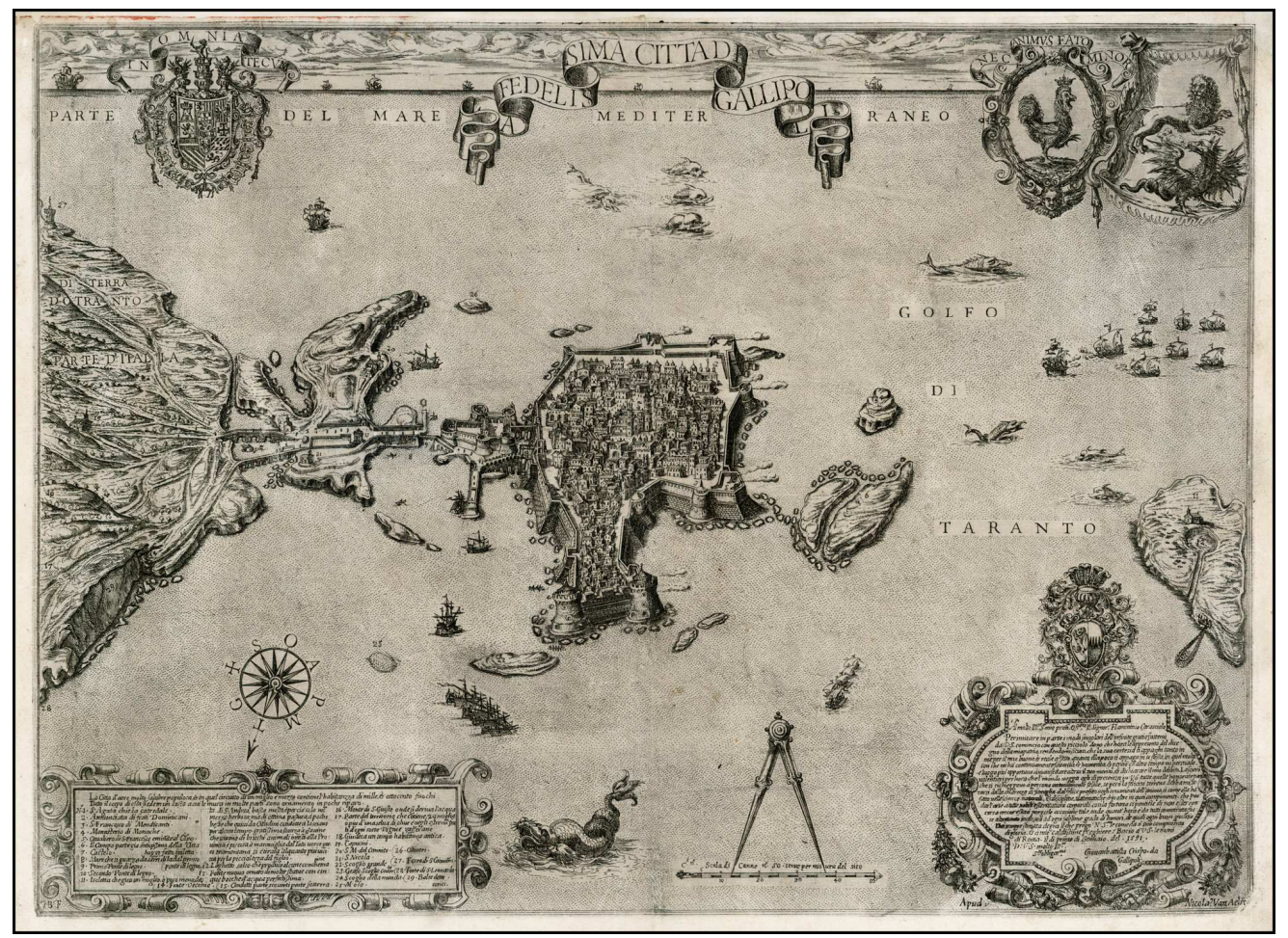

Fig. 4. La rappresentazione di G.B. Crispo (1591).

\section{Cenni storici}

Gallipoli è una cittadina che insiste sulla costa

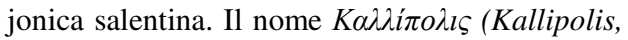
"Città bella", di diretta derivazione greca), venne attribuito con tutta probabilità ad un abitato portuale preesistente che una tradizione mai del tutto convalidata denomina invece Anxas ${ }^{5}$, connesso con la vicina città messapica di Alixias (Alezio). E' certo che alla metà del VI secolo Gallipoli è sede vescovile e dipendeva amministrativamente da Bisanzio, nell'orbita della quale rimase con Otranto anche durante l'occupazione longobarda. Alla fine del IX secolo Gallipoli divenne strategicamente importante per il controllo delle rotte ioniche ed è documentato che vi affluirono coloni greci; di questa fase è il nucleo più antico del castello e da questo periodo la struttura urbanistica della città rimane funzionalmente inalterata. Le notizie rimangono scarse sino al 1269, quando gli Angioini devastarono la città per la sua nota fedeltà alla causa sveva, a tal punto che il vescovo trasferì la sua sede nella vicina Alezio. Del XIV secolo è la ricostruzione in forme gotiche, quasi al centro dell'abitato, della Cattedrale.

Afflitta dalla peste nel 1429, dallo sbarco di galeotte turche (20 aprile 1430), e dalla devastazione delle truppe aragonesi, passata a Ferdinando I d'Aragona e fortificata, resisté alle forze turche invadenti Terra d'Otranto (1481) e ai Veneziani, che l'espugnarono nel maggio del 1484.

A metà del XV secolo la città conta solamente 160 fuochi, a fronte dei 540 fuochi della vicina Nardò; il Galateo nella sua Callipolis descriptio (1512-1513) parla di una città fiorente e fortissima, dilungandosi sulla sua particolare struttura urbana articolata in percorsi tortuosi, appositamente predisposti per contrastare l'impetuosità dei venti più molesti. Nel 1532 si contano 988 fuochi; nel 1545 con 1131 fuochi, Gallipoli è il secondo centro della provincia dopo Lecce. È in questo periodo si adeguano alle nuove tecniche militari le mura e il castello, si insediano nuovi ordini religiosi (Clarisse, Cappuccini, e Paolotti) e si compie la definitiva trasformazione 
dell'abitato medievale. Alla fine del secolo XVI Gallipoli conta 1700 fuochi contro i 1285 di Nardò (Cazzato, et al., 2015).

\section{La pianta di Crispo}

Addì primo di gennaio del 1591, il Gallipolino Giovan Battista Crispo $^{6}$ dedica al suo mecenate Il disegno della mia patria inciso da Nicolò Beatrizet e stampato da Nicola Van Aelst.

L'incisione rappresenta con ricchezza di particolari il nucleo urbano ed ha goduto di grande fortuna, essendo stata riproposta con minime variazioni in numerose pubblicazioni successive ${ }^{7}$; la legenda fornisce molti altri utili particolari.

"La Città d'aere molto salubre populosa, e in quel circuito di un miglio e mezzo contiene l'habitanzza di mille, e ottocento fuochi. Tutto il corpo di essa siede in un sasso a cui le mura in molte parti sono ornamento in poche riparo."

La città è collegata da un ponte ligneo al castello, che a sua volta è collegato alla terraferma con un ulteriore ponte di legno. La mappa mostra il "fonte nuovo ornato di molte state con cinque bocche d'acqua perfectissima". L'acqua è approvvigionata da "Condotti parte scoverti parte sotterra" che si collega al "Fonte vecchio", alimentato dal "Monte di S. Giusto onde si deriva l'acqua".

Le mura raffigurate nella mappa evidenziano un secondo livello che, impostato sulla la parte scarpata, si erge sul piano viario e quasi sormonta le costruzioni. Tale rappresentazione concorda con quella di Piri Re'is: il particolare non è tuttavia indicativo, in quanto tale rappresentazione è comune a tutte le città fortificate disegnate nel Kitab i bahriye.

La maggiore altezza delle fortificazioni originarie è comunque confermata dai pochi tratti rimasti (Fig. 5); il De Giorgi in proposito così scriveva (Giorgi, 1882): "Il perimetro delle mura di Gallipoli gira per circa un chilometro e mezzo. In questi ultimi anni si è però abbattuta quella parte che sporgeva sul piano dell'isola [...]. Gallipoli non è più la Urbs munita et rupibus circumsepta, et firmissimis muris del Galateo; ma in compenso ci ha guadagnato moltissimo l'igiene delle vie e la ventilazione delle case. I palazzi e le chiese, che sembravano affogati dietro quegli alti baluardi, sono venuti fuori tutti lindi e puliti a specchiarsi nello Jonio, ed una larga via oggi circonda la città."

Un altro particolare degno di rilievo della pianta è la presenza della Porta Terra, unico accesso alla Città, risalente al 1310 e demolita insieme alle mura tra il 1879 e il 1887 , munita di una sorta di belvedere descritto dallo storico locale Bartolomeo Ravenna?.

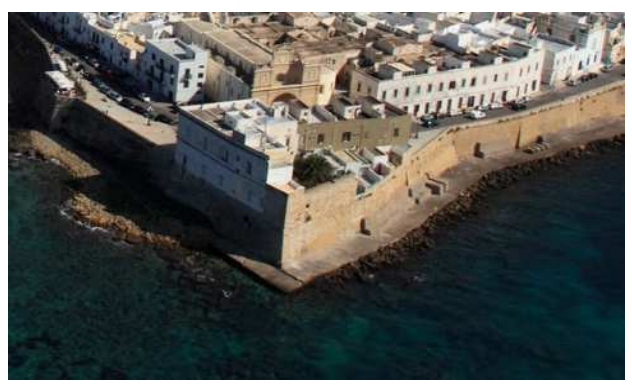

Fig. 5. Tratto superstite di mura (lato ovest) che ha conservato l'altezza originaria.

\section{Le rappresentazioni successive}

Il Theatro delle città d'Italia pubblicato nel 1616 da Pietro Bertelli, libraro in Padova, riporta una mappa che è con ogni evidenza derivata da quella del Crispo, cui nulla aggiunge. Rimarchevole è la circostanza che Gallipoli sia l'unica città pugliese, oltre Taranto, che compare nell'opera. Nella descrizione della città il Bertelli riporta le notizie già fornite dal Crispo nella sua mappa, quali ad esempio la lunghezza del circuito murario e il numero dei fuochi; aggiunge che "Ella è sì come un luogo attissimo alli mercati, perché li mercatanti vi possono d'ogni luogo venire tanto per acqua, quanto per terra. Et pertanto da ogni stagione dell'anno vi si veggono mercatanti, e massimamente genovesi. [...] Ha questa città buono, e fertile paese, dal qual si cava assai oglio, vino, zaffarano, e altre cose per il bisogno de gli huomini, con gran moltitudine di pesci" (Bertelli, 1616).

La descrizione introduce le attuali e soprattutto prefigura le future fortune di Gallipoli come centro di commerci. A cavallo tra il XVI e XVII secolo risale "lo sviluppo del traffico dell'olio, 


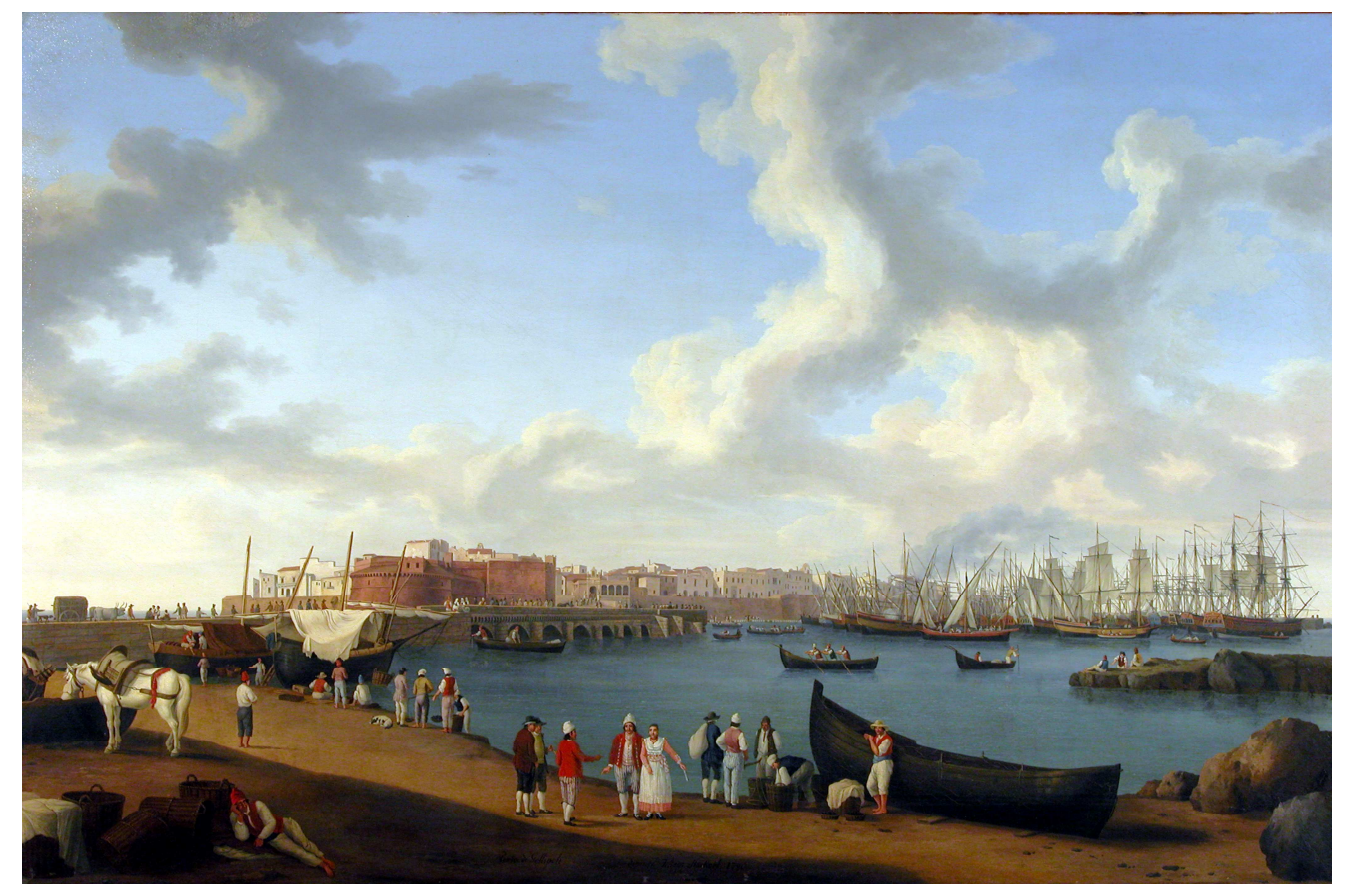

Fig. 6. Porto di Gallipoli di Jakob Philipp Hachert.

attività preponderante della città che da tempo immemorabile godeva anche del diritto di stabilirne il prezzo" (Saladini, 1980).

L'importanza commerciale del porto di Gallipoli è confermato dall'opera di Jakob Philipp Hackert (1737-1807), pittore di corte di Ferdinando IV di Borbone, che tra il 1789 e il 1792 riproduce su tele di grandi dimensioni i maggiori porti delle province orientali del Regno di Napoli.

Nel 1836, quando Bartolomeo Ravenna scrive la sua opera Memorie istoriche della città di Gallipoli, corredata dalla planimetria dettagliata del centro urbano che ci restituisce ancora l'immagine della città compresa nell'originario circuito difensivo cinquecentesco, da questa descrizione dello sviluppo raggiunto dalla cittadina: “[...] l'abbondanza della pesca, de' vegetabili, delle frutta, del vino, delle legna, dell'olio, e di tutto ciò, che principalmente abbisogna la vita umana, giunge ad un grado soddisfacente. Il commercio ne ha accresciuto il pregio. La popolazione, compreso il territorio, è al di sopra dei novemila abitanti. Quei che dimorano nella Città non si occupano all'agricoltura, ma solo al commercio, alle arti ed alla pesca".

Lo sviluppo commerciale e demografico raggiunto nel corso del XVIII secolo non era più compatibile con la struttura urbana compresa tra le mura della città vecchia.

Già nel 1783 la comunità richiese all'autorità centrale l'ampliamento urbano non più differibile, ed impedito dalla presenza, sia nella città antica, che sulla terraferma, di vincoli e servitù militari.

Il vincolo di rispetto militare si estendeva al di là del ponte di accesso sulla terraferma per 330 tese (circa $695 \mathrm{~m}$ ): al di là del ponte esistevano solo una fabbrica di botti, la fontana greca, la cappella di S. Cristina, la Chiesa del Canneto e i magazzini della tonnara, come si vede ancora nel 1836 nella mappa del Ravenna (Fig. 7).

L'amministrazione Borbonica inviò per lo scopo l'ingegnere gallipolino Vincenzo Ferrarese, che già stava lavorando in Calabria per la ricostruzione dei borghi periti nel terremoto del 1783 . 


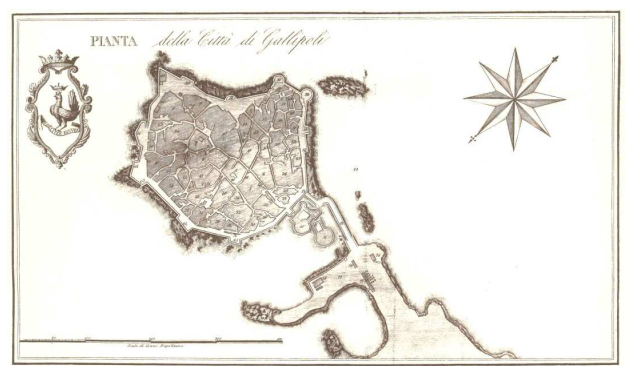

Fig. 7. Bartolomeo Ravenna, Memorie istoriche della città di Gallipoli, Napoli, 1936. La mappa dimostra che agli inizi del XIX secolo la città di Gallipoli è ancora compresa nell'originario perimetro cinquecentesco.

Sebbene nel 1785 lo stesso Ferrarese avesse personalmente sovrainteso allo sbancamento dei terreni interessati dal progetto di espansione, l'autorizzazione regia intervenne solo nel 1835 , e la concessione del primo lotto edificabile data solo al 1858 [Saladini, 1980]. Si assiste quindi alla lenta trasformazione della città che abbandona i limiti urbanistici dell'isola-fortezza e si espande sulla terraferma con l'edificazione del Borgo; un assetto planimetrico con larghe vie rettilinee che si intersecano ad angolo retto.

Negli anni Quaranta del secolo scorso il Borgo può dirsi completato.

\section{Conclusioni}

Nel 1897 l'esportazione di olio di oliva dal Porto di Gallipoli veniva valutata in 7000 tonnellate e il movimento del suo porto in circa 1500 unità tra velieri e piroscafi (Saladini, 1980). La storia del dopoguerra è caratterizzata dal progressivo venire meno dell'importanza economica e commerciale della città e del porto a causa delle mutate condizioni economiche, che hanno visto il progressivo abbandono delle tradizionali attività agricole e manifatturiere su cui Gallipoli ha per secoli fondato la sua prosperità.

Lo sviluppo dei giorni nostri è legato alla pesca ed allo sfruttamento turistico del territorio che si è concentrato principalmente sulla costa mettendo a rischio i valori paesaggistici e l'assetto territoriale conservatosi praticamente integro nel corso dei secoli.

\section{Note}

1 Ahmet Muhiddin Piri, conosciuto come Piri Re'is, è una singolare figura di uomo d'arme, di mare, di studio turco, nato a Gallipoli sui Dardanelli (1465-1470) e giustiziato in Egitto a 85 anni perchè accusato di tradimento. Nipote dell'ammiraglio turco Kemāl Re'îs, fu capitano navale ottomano e in seguito ammiraglio ( $\operatorname{Re}^{\prime} \overline{\mathrm{i}} \mathrm{s}$ significa "comandante"). A lui dobbiamo una "mappa del mondo", oggi conservata nel museo del Topkapi, disegnata partendo da materiali spagnoli e portoghesi e datata 1513.

${ }^{2}$ L'argomento principale del Libro del mare sono le coste e le isole del Mar Mediterraneo. Dopo una parte introduttiva scritta in versi e che illustra in generale $\mathrm{i}$ vari argomenti che attengono alla navigazione, la parte principale del libro consiste di 743 fogli e comprende 215 mappe e carte. Questa seconda parte, a differenza della prima, è scritta in prosa, ed illustra porti e città costiere del Mediterraneo.

${ }^{3}$ Una incerta tradizione fissa la data di fondazione del complesso al 1217; tuttavia la data documentata di fondazione risale al XV secolo.

${ }^{4}$ Il chiostro dei Domenicani conserva ancora resti dell'originaria struttura cinquecentesca; l'attigua chiesa fu completamente ricostruita agli inizi del XVIII secolo. La chiesa confraternale del Carmine risulta ricostruita nel 1838 sul luogo in cui, fin dal 1530, è attestata la presenza dell' antico tempio.

${ }^{5}$ Plinio il Vecchio nella Naturalis historia, fa cenno a Callipolis, quae nunc est Anxa.

${ }^{6}$ Giovan Battista Crispo nacque a Gallipoli nel 1550. Si recò a Napoli dove seguì studi di filosofia ed entrò in contatto con varie personalità del suo tempo. A seguito dei rovesci economici del padre, fu precettore, tra l'altro in casa di Flaminio Caracciolo al quale dedicò la rappresentazione prospettica di Gallipoli incisa da Nicola Beatrizet.

7 Tra le più pregevoli, l'incisione su rame del 1598 con eccellente coloritura coeva pubblicata da Georg Braun (1541-1622) autore della Civitates orbis terrarum che pubblicò in collaborazione con il cartografo Franz Hogenberg (1535- 
1590) a Colonia, e quella dell'Atlante dell'Hondo Jodoco, Leida 1625.

${ }^{8}$ Il miglio napoletano, stabilito con regio Decreto del 1480 , misura $1845,69 \mathrm{~m}$ circa; se ne deduce che il perimetro dell'isola è pari, secondo il Crispo, a $2768 \mathrm{~m}$; la misura reale del perimetro, incluso il castello, è circa $2000 \mathrm{~m}$.
9 “Entrando per 1'unica porta, trovasi sulla dritta un luogo elevato che si nomina il Seggio. Questo è di varj archi di fabbrica con covertura di legno, ove coloro a' quali piace di trattenercisi, godono comodamente la vista del porto de' bastimenti ancorati, del caricamento, delle campagne, del cratere verso tramontana, della pianura e delle colline, che le fanno corona".

\section{Bibliography}

Bertelli, P. (1616). Il Theatro delle città d'Italia, Padova.

Cazzato, V.; Cazzato, M., coord. (2015). Atlante del Barocco in Italia. Lecce e il Salento. Vol. 1. I centri urbani, le architetture e il cantiere barocco, De Luca Editori d'Arte, Roma, pp. 231-243.

Coppola, G.; Colamonico, C.; Ciasca, R.; Manfroni, C. (1932). Gallipoli, Voce dell'Enciclopedia Italiana Treccani.

Giorgi, C. De. (1882). La Provincia di Lecce. Bozzetti, Congedo Ed., Galatina, pp. 55-64.

Mario, M. De. (2002). Gallipoli. Guida storica ed artistica, Capone Ed.

Piri Re'is. (1520). Kitab i bahriye, The Walters Art Museum, 600 N, Charles Street, Baltimore, Maryland 21201.

Polito, A. (2015). Giovan Battista Crispo, l'illustre gallipolino che, secondo Wikipedia, avrebbe trovato e salvato a Napoli l'Arcadia del Sannazzaro, in http://www.fondazioneterradotranto.it.

Saladini, C.M, (1980). "Gallipoli”, in Storia dell'arte italiana. Inchieste su centri minori, Giulio Einaudi Editore, pp. 341-363.

Silvestri, F. (1986). Imago Apuliae. Geografia e immagini della Puglia nella cartografia storica storica italiana ed europea, Capone Ed., pp. 79-148. 
